\title{
Correlación entre estado nutricional materno, calidad de la lactancia y crecimiento del niño*
}

\author{
Eduardo Atalah,** Patricia Bustos, ** Manuel Ruz,** Car- \\ men Hurtado,"*** Lilia Masson, ${ }^{* * * *}$ Carmen Urteaga ${ }^{* *}$ \\ Magda Castaños, ${ }^{* *}$ Rousana Godoy, ${ }^{* *}$ Humberto Olj- \\ ver*** y Julia Araya.**
}

\begin{abstract}
With the purposes in study the possible relationships among nutrition and molher's alimentation, quality of the milk and growth of the infant, 14 thin nurses were studied during the first months of lactation. The control group was forned by 20 nornal nurses.

The analysis of the diet was made for quantified dietary bistory. The results indicated thal the calorie intake was deficient in $\mathbf{3 0 . 8 \%}$ and $20.0 \%$ for the thin and Control group respectively. This value increased to $35 \%$ when one day recall dietary survey was considered. Also il was detected a similar value of mother with deficient protein intake.

At the 3 month of lactation the sons of the thin mothers bad the weight, height and arm perimeter significanty lesser than the sons of cuntrol's mothers. Alhough these differences both groups shown sinilar increments accordins to international standards.

Chemical composition of the milk showed nut significatives differences between Conlrol and thin groups for any of the nutriments analyzed, all of them were into the sverage values of the develogped councries.

To relate infsnt's weight at the 3 munths with the variables in study a sigaificative correlation was found with the birth weight, fat caloric and linoleic acid content in the milk. The unilk composition had hot correlation with any of the materral indicator analyzed.

The results guagest that the differences found in infant's growih of bolh groups of mothers depends more of the volume that of the composilion of the milk. Prubably it would be more important the nutritional status of the mother prior the breast feeding period than the alimentation received during it for to get a succesaful lactation. In urden to confirm these findings it is necessary to realize experiences with dietary suplements for to try to reduce morbility and mortality risks in the first period of the life.
\end{abstract}

La importancia de la lactancia natural en las primeras etapas de la vida ha sido suficientemente enfatizada en los últimos años a través de numerosos estudios. ${ }^{(1,5)}$ Es un hecho conocido también que existe a nivel mundial una fuerte tendencia al descenso de esta práctica, evidenciada tanto en países desarrollados como aquellos en vias de desarrollo. ${ }^{\text {(6, }, ~}$ ") $\mathrm{La}$ forma habitual de enfrentar esta situación ha sido a través de programas educativos, ${ }^{10,12)}$ sin que generalmente se consideren aspectos nutricionales, que pueden afectar el éxitu de la lactancia.

Es fácil de entender y aceptar los mayores requerimientos nutricionales generados por la lactancia. No obstante, aủn está en discusión si una alimentación deficiente o una disminución de las reservas con que la madre inicia la lactancia son capaces de alterarla. La información que se dispone con respecto a este hecho es contradictoria y dificil de inter-

\footnotetext{
* Financiado parcialmente por el Servicio de Desarrollo Cientítico, Artístico y de Cooperación Intemacional, U, de Chile. Proyectos 458-791 y $141-742$.

*"Departamento de Nutrición. Facultad de Medicina Santiago Norte, U. de Chile.

*" "Departamentu de Química y Tecnología de los Nimentua, Facultad de Cienciar Quínicas, U. de Chile.
}

pretar. Se han comunicado diferencias en volumen, aporte energético, cantidad y calidad de ácidos grasos, vitaminas lipo e hidrosolubles en la leche de nodrizas "bien" y "mal" alimentadas o provenientes de distinto nivel socioeconómico. ${ }^{13,20)}$ Sin embargo, dado que en los estudios se han aplicado métodos de muestreo y análisis y que no siempre se ha definido el estado nutriciunal materno, es difícil llegar a conclusiones definitivas.

Por otra parte, es importante conocer el efecto del estado nutricional sobre la lactancia en nuestro medio, donde la nodriza se encuentra protegida por programas de suplementación alimentaria y donde los déficits extremos de otros paises no son frecuentes. Basados en esto, nos propusimos analizar la relación entre nutrición materna, composieión química de la leche y crecimiento del lactante durante el primer trimestre de la vida, en un grupo de nodrizas del Area Norte de Santiago.

\section{MATERIAL Y METODO}

Se seleccionó en la Maternidad del Hospital José Joaquín Aguirre 41 puérperas, con embarazo de término, que no presentaron patología materna ofetal que afecten el crecimiento. Fueron clasificadas 
desde un punto de vista antroponétricos, en dos grupos:

- 25 contcoles con indice peso-talla, ${ }^{(2)}$ perímetro braquial y/o pliegue tricipital superior al $100 \%$ del estándar respectivo. ${ }^{\text {(2) }}$

- 16 entlaquecidas, con índice peso-talla y/o perímetro braquial y pliegue tricipital inferior al $90 \%$ del estándar.

Ambos grupos fueron posteriormente visitados en domicilio a las 4-8 y 12 semanas postparto y en condiciones estandarizadas se estudió a la madre y el niño.

a) Madre:

-Encuesta socioeconómica segín Graffar. ${ }^{\text {(23) }}$

- Encuesta alimentaria por tendencia de consumo cuantificada ${ }^{(24 !}$ y recordatorio de 24 horas. ${ }^{(25)}$

- Medición de peso corporal, talla, perímetro braquial y pliegue cutáneo tricipital, bicipital, subescapular y suprailíaco, a partir de los cuales se calculó perímetro muscular braquial ${ }^{22)}$ y masa grasa. ${ }^{\{20\}}$

- Muestra de leche por vaciamiento manual completo de un pecho, en la que se determinó grasa total según el método recomendado por $\mathrm{AOAC} ; 2 * 1$ N total y no proteico por micro-Kjeldahl;'28) lactosa por una adaptación del método de Somogyi Nelson ;29) $^{20}$ engia aplicando los factores $8.79,4.27$ y 3.87 cals. /g. para grasa, proteina y lactosa, respectivamente; acido linoleico por cromatografía de gas líquido del ester metílico ${ }^{130 !}$ y calcio por espectrofotometría de absorción atómica. ${ }^{\{31\}}$

\section{b) Lactante:}

- Medición de peso corpiral, talla total y sentado, perímetro craneano, torácico, del brazo y del muslo; longitud del brazo y antebrazo y pliegues cutáneos tricipital y subescapular, en base a los cuales se estimó la masa grasa según Dauncey. ${ }^{\text {(32) }}$

El aporte de nutrientes de la encuesta alimentaria fue calculado utilizando la tabla de composición química de alimentos nacionales ${ }^{(33)}$ y del Instituto de Nutrición de Centroamérica y Panamá. ${ }^{(3)}$ Para estimar la adecuación de la ingesta se utilizaron las recomendaciones FAO/OMS para la nodriza, ${ }^{135 .}{ }^{361}$ estimándose como deficiente una cobertura inferior al $75 \%$. Se determinó el nivel seguro de ingesta de proteínas considerando score 70 y digestibilidad 85 .

Se retiraron del estudio aquellas madres que iniciaron alimentación láctea artificial, de modo que el grupo definitivo, a las 4, 8 y 12 semanas quedó constituido por 20,20 y 16 controles y 14,14 y 9 enflaquecidas, respectivamente.

En el análisis estadístico se utilizó la prueba "t" de Student para diferencia de medias, análisis de correlación y regresión. Fueron consideradas como significativas las diferencias como un $\mathrm{p}<0.05$. $^{\mid 3 i ।}$

\section{RESULTADOS}

Algunas características generales y antropométricas de las nodrizas al inicio de la lactancia se muestran en Tabla 1. Desde un punto de vista nutricional, hay claras diferencias entre ambos grupos en todos los indicadores utilizados ( $p<0.01$ ), a excepción de la

\section{Tabla 1}

Características generales y antropométricas de las madres al inicio de la lactancia

\begin{tabular}{lrcr}
\hline Indicador & $\begin{array}{c}\text { Controles } \\
\overline{\mathrm{X}}\end{array}$ & $\begin{array}{c}\text { Enflaquecidas } \\
\overline{\mathrm{X}}\end{array}$ & $\mathrm{P}$ \\
\hline Edad años & 28.7 & 21.1 & $<0.001$ \\
Paridad & 3.2 & 2.3 & $\mathrm{~N} . \mathrm{S}$. \\
Puntaje socioeconómico & 18.6 & 19.3 & N. S. \\
Talla cm. & 155.3 & 154.8 & N. S. \\
Relación peso-talla \% & 124.4 & 87.8 & $<0.001$ \\
Masa grasa \% & 31.8 & 21.7 & $\ll 0.001$ \\
P. musc. braquial cm. & 23.1 & 19.4 & $\ll 0.001$ \\
\hline \multicolumn{1}{c}{ Número } & 20 & 14 & \\
\hline
\end{tabular}


talla. El grupo contrul presenta una relación pesotalla y masa grasa sobre lo normal, lo cual se espera en la puérpera, por el depósito de tejido graso adquirido durante el embarazo. ${ }^{\text {(35t }} \mathrm{El}$ grupo enflaquecido, que en general incluye madres de menor edad que el grupo control $(p<0.001$ ), muestra déficit antropométricos que pueden considerarse leves a moderados. El nivel socioeconúmico promedio corresponde al grupo IV o medio bajo de la escala de Graffar.

La ingesta promedio-día de energía y algunos nutrientes calculada sobre la base de la encuesta por tendencia de consumo cuantificada se muestra en Tabla 2. En general, están dentro de un nivel aceptable, llamando la atención que es superior en el grupo enflaquecidas, aun cuando las diferencias no alcanzan sígnificación estadística. Dada la gran variabilidad en la ingesta, se hace necesario analizar no sölo el promedio, sino también el grado de cobertura individual a la recomendación FAO/OMS, presentándose en Tabla 3 el porcentaje de madres con ingesta deficiente $\{\leqslant 75 \%$ ). Destaca la moderada frecuencia de déficit energético y en menor proporción de proteínas, lo que se observa preferentemente en el grupo enflaquecidas, sin que llegue a ser estadísticamente significativo.

Llama la atención que cerca de la mitad de las madres tiene una baja ingesta de calcio. Este hecho ha sido señalado también en otras edades, ${ }^{\mid 33]}$ siendo su importancia discutible, dado lo imprecisa que puede estimarse la recomendación y la ausencia de patología atribuible a déficit de este nutriente.

Con el objeto de implementar la información alimentaria, se realizó una encuesta por recordatorio de 24 horas, que para algunos autores es más confiable. Esta encuesta indicó para todos los nutrientes ingestas inferiores a la tendencia de consumo. A comparar las dos encuestas entre sí, la diferencia
Tabla 3

Porcentaje de nodrizas con ingesta $\leqslant 75 \%$ de la recomendación FAO/OMS

\begin{tabular}{lccc}
\hline Nutriente & \multicolumn{2}{c}{ Ingesta deficiente } & $P$ \\
\cline { 2 - 3 } & $\begin{array}{c}\text { Controles } \\
\%\end{array}$ & $\begin{array}{c}\text { Enflaquecidas } \\
\%\end{array}$ & \\
\hline Energía & 20.0 & 30.8 & N.S. \\
Proteinas & 10.0 & 23.1 & N.S. \\
Calcio & 50.0 & 46.2 & N.S. \\
\hline
\end{tabular}

promedio fue de 355 calorias (14\%), proteinas $18.3 \mathrm{~g}$. (21\%) y calcio $258 \mathrm{mg}$. (25\%), con un comportamiento muy semejante en controles y enflaquecidas, Considerando esta última encuesta, el déficit calórico o pruteico afecta aproximadamente a un $35 \%$ de las nodrizas en ambos grupos. La alta proporción de casus con déficit de ingesta proteica es una situación no observada en otros grupos etáreos. ${ }^{\text {3a, 39) }}$

Algunos indicadores antropométricos del lactante a las 12 semanas de vida se presentan en Tabla 4. Destaca el hecho de que los hijos de nodrizas enflaquecidas muestran valores estadísticamente inferiores en peso, talla y perímetro braquial en relación al grupo control. Aproximadamente el 50\% de esta diferencia está determinada al nacimiento y el resto por una menor velocidad de crecimiento posterior, reflejando el efecto de la nutrición materna tanto en el embarazo como en la lactancia. ${ }^{(4), 4)} \mathrm{Sin}$ embargo, es importante recalcar, como hemos comunicado previamente, que a pesar de la desnutrición materna se logra con la lactancia exclusiva una

Tabla 2

Ingesta promedio-día de energía y algunos nutrientes según encuesta de tendencia de consumo cuantificada

\begin{tabular}{|c|c|c|c|}
\hline Ingesta/día & $\underset{\overline{\mathbf{X}}}{\text { Controles }}$ & ${ }_{\overline{\mathrm{X}}}$ Enflaquecidas & $\mathbf{P}$ \\
\hline Energía Kcal. & 2.477 & 2.707 & N.S. \\
\hline Proteínas g. & 83.5 & 92.1 & N.S. \\
\hline Grasa g- & 64.9 & 66.8 & N.S. \\
\hline Calcio mg. & 995 & 1106 & N.S. \\
\hline
\end{tabular}




\section{Tabla 4}

Características antropométricas del lactante de 12 semanas según estado nutricional materno

\begin{tabular}{lccc}
\hline Indicador & $\begin{array}{c}\text { Controles } \\
\overline{\mathrm{X}}\end{array}$ & $\begin{array}{c}\text { Enflaquecidas } \\
\overline{\mathrm{X}}\end{array}$ & $\mathrm{P}$ \\
\hline Peso g. & 6.253 & 5.621 & $<0.05$ \\
Talla cm. & 60.5 & 59.0 & $<0.05$ \\
Perímetro braquial cm. & 13.7 & 12.6 & $<0.01$ \\
Masa grasa \% & 29.0 & 26.5 & N.S. \\
\hline
\end{tabular}

excelente evolución pondoestatural, que se compara satisfactoriamente con patrones internacionales. ${ }^{(4 i, 4)}$

En cada nodriza que continuó con lactancia exclusiva se obtuvo una muestra de leche a las 4,8 y 12 semanas. La composición centesimal promedio para el total de muestras obtenidas en cada grupo se muestra en Tabla 5. Ninguno de los parámetros estudiados señala diferencias estadísticas entre ambos grupos. Contrariamente a lo esperado, Jas grasas no variaron en cantidad ni calidad en el grupo enflaquecidas respecto al control. En los dos grupos este nutriente fue el que evidenció una mayor variabilidad, con un rango que fluctuó entre 0.9 y $9.0 \mathrm{~g} . / \mathrm{dl}$. El contenido de grasa en una misma madre a través del tiempo tampoco fue constante, existiendo diferentes

Tabla 5

Composición centesimal de la leche en nodrizas de diferente estado nutricional

\begin{tabular}{lccc}
\hline Nutriente & $\begin{array}{c}\text { Controles } \\
\overline{\mathrm{X}}\end{array}$ & $\begin{array}{c}\text { Enflaquecidas } \\
\overline{\mathrm{X}}\end{array}$ & $\mathrm{P}$ \\
\hline Energía Cal/dl & 65.2 & 63.5 & N.S. \\
Proteína g/dl & 1.0 & 1.1 & N.S. \\
Grasa g/dl & 4.0 & 3.7 & N.S. \\
Lactosa g/dl & 6.7 & 6.9 & N.S. \\
$\begin{array}{l}\text { Calcio mg/dl } \\
\text { Ac. linoleico } \\
\text { peso \% }\end{array}$ & 29.2 & 31.0 & N.S. \\
\hline \multicolumn{1}{c}{ Número* } & $\mathbf{5 5}$ & 15.3 & N.S. \\
\hline
\end{tabular}

*Incluye muestras de 4,8 y 12 semanas de lactancia. tendencias, como se muestra en tres casos extremos en el Gráfico 1. Parte de la diferencia podría explicarse por variaciones en la extracción de la muestra, ${ }^{(43)}$ aun cuando se trató de estandarizar al máximo la técnica.

Otro hecho que destaca es que todos los nutrientes estudiados se encuentran dentro de los rangos habituales para madres provenientes de países desarrollados o de buen nivel de vida. ${ }^{(15,17,44,461}$ Hace excepción el ácido linoleico, que analizado en mg./dl., o como porcentaje de las grasas o calorías totales, se encuentra por encima de lo comunicado por otros autores. ${ }^{(17,47,48)}$

En las 25 madres que completaron 3 meses de lactancia exclusiva se realizó un análisis estadístico de regresión lineal considerando como variable de-

$$
\text { I RAFICO } 1 \text {. }
$$

VARIACION DEL CONTENIOO DE GRASA DE LA LECHE EN 3 NODRIZAS NORMALES

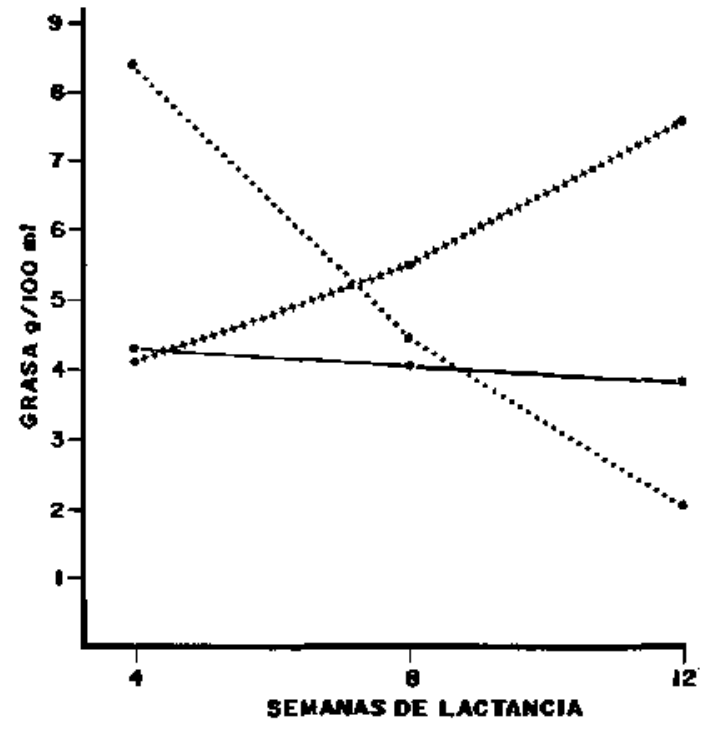


pendiente el peso del lactante a los 3 meses y como variable independiente los otros indicadores estudiados. Se presentan en Tabla 6 los coeficientes de correlación obtenidos, destacando que el más alto se da en relación con el peso de nacimiento, el que a su vez se ha demostrado claramente asociado con el

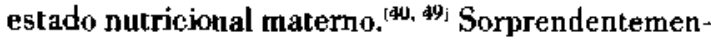
te el nivel socioeconómica se correlaciona en forma inversa con el peso del lactante alimentado al pecho (a menor nivel, mayor peso), lo que no estaría condicionado por variables nutricionales o alimentaries maternas ni por la composición quimica de la leche. Una posible explicación puede estar en diferentes prácticas en cuanto a la lactancia en los grupos de menores ingresos en relación al nivel socioeconómico medio. La relación peso-talla, la masa grasa materna, el contenido de grasas y de ácido linoleico de la leche se correlacionaron positjvamente con el peso del lactante. Sin embargo, las variables abmentarias como adecuación de la ingesta calórica, proteica o un indicador en base a ambas no demostraron asociación con el peso. Esto sugiere que más importante que la alimentación durante la lactancia es el estado nutricional durante el embarazo, lo que condiciona el peso de nacimiento y las reservas (pesotalla, masa grasa) con que la madre inicia la lactancia.

\section{Tabla 6}

Coeficiente de correlación entre peso del lactante a los 3 meses y las variables analizadas

\begin{tabular}{lcc}
\hline \multicolumn{1}{c}{ Variable } & $\begin{array}{c}\text { Coeficiente } \\
\end{array}$ & $\mathrm{P}$ \\
\hline Peso nacimiento & 0.58 & $<0.001$ \\
Puntaje socioeconómico* & 0.47 & $<0.02$ \\
Grasa láctea & 0.46 & $<0.02$ \\
Peso-talla materno & $0.42<0.05$ \\
Masa grasa materna & $0.42<0.05$ \\
Ac. linoleico lácteo & $0.40<0.05$ \\
P. musc. braquial materno & 0.31 & N.S. \\
Ingesta calórico-proteica & 0.10 & N.S. \\
\hline
\end{tabular}

PPuntaje indica $<$ N.S.E.

Un análisis semejante se realizó considerando como variable dependiente el contenido calórico, graso y de ácido linoleico de la leche materna. Ninguna de las variables estudiades (antropométricas, alimentariag y socioeconómicas) se asoció con la composición química de la leche. Dentra del rango estudiado, la calidad de la leche es independiente de factores nutricionales maternos.

\section{DISCUSION}

Las relaciones entre nutrición materna y lactante continúan siendo objeto de discusión. La información que se dispone sugiere diferencias en volumen de leche secretado y concentración de algunos nutrientes en leche de madres de diferente estado nutricional, aun cuando otros autores no han logrado demostrarlo. Esta inconsistencia puede ser parcialmente explicada por dos hechos:

a) Deficiente definición de las condiciones nutricionales maternas. La mayor parte de los estudios se basan en datos de comunidades pobres, o "mal alimentadas", asumiéndose que pobreza es sinónimo de desnutrición, lo cual no siempre puede ser efectivo.

b) Diferentes grados de compromiso nutricional de las poblaciones estudiadas. En ese sentido no son comparables las nodrizas analizadas por Edoxien en Nigeria ${ }^{(30)}$ o Lindbland en Pakistán ${ }^{(511}$ a las madres de nuestro grupo, las que en función del déficit alimentario o antropométrico deben ser clasificadas sólo como desnutrición leve o moderada. Es posible que mecanismos de adaptación permitan mantener una composicion constante de la leche frente a un déficit nutricional discreto, pero no cuando las condiciones son extremas.

Aun cuando nuestros grupos son claramente diferentes desde el punto de vista antropométrico, no encontramos madres con una importante emaciación muscular a de tejido graso. Sorprendentemente la ingesta alimentaria fue comparable y aun superior en las enflaquecidas y en ambos grupos mayor a lo observado en otros países en vías de desarrollo. ${ }^{\text {(52) }}$ Como hemos comunicado previamente, ${ }^{41)}$ los cambios antropométricos de la madre durante la lactancia fueron mínimos, lo que validó en cjerta forma los datos de ingesta alimentaria.

El crecimiento del lactante fue significativamente menor en el grupo de madres enflaquecidas, tanto en peso, talla como en perímetro braquial. La diferencia está originada en parte por el peso de nacimiento y en parte por una menor velocidad de crecimiento posterior, reflejando la importancia de la nutrición en el embarazo y lactancia. A pesar de las diferencias, el incremento del grupo enflaquecida fue absolutamente comparable al P50 de las tablas 
de Boston o del NCHS e incluso superí en $273 \mathrm{~g}$. el promedio de Sempé. Queda por establecer durante cuánto tiempo es posible mantener una adecuada evolución pondoestatural. El reducido número de lactantes que continuó lactandu impidis obtener conclusiones, pero según numerosos autores es de esperar una disminución del crecimiento entre el $4 .{ }^{\circ}$ y 6." mes, cuando la situación nutricional de la madre no es satisfactoria. ${ }^{\langle 15,5,5\}}$

La composición de la leche, en relacion a lus macronutrientes estudiados, no se vio modilicada por las caracteristicas nutricionales de la madre. Esto contirma el hecho de que la nodriza mantiene la capacidad de secretar leche en calidad constante, a pesar de restricciones nutricionales moderadas. Aun cuando en los primeros meses de lactancia el contenido prumedio de grasas en el grupo enflaqueeidas fue $6.5 \mathrm{~g} . / \mathrm{dl}$. menor que el control. no alcanzó significación estadística, dada la gran variabilidad en ambos grupos.

La diferencia en el arecimiento del niño al no ser explicado por cambios en la calidad de la leche, debe buscarse en diferencias de la cantidad secretada. En relación a este aspecto, Hanafy demistri en nodrizas semejantes a las de este estudio, que el apurte tutal diario de nutrientes al lactante disminuía significativamente en madres desnutridas por disminución del volumen de leche. ${ }^{|\zeta|}$

Es importante validar estos resultados, ya que se podria mejorar la lactancia aumentando la asistencia nutricional en aquellas madres que lo requieran. Lo lógico debiera ser suplementar a la nodriza antes que introducir alimentaciun artificial al niño, con todos los riesgos que ello implica. Sin embargo, nustros propios resultados nos demuestran que más importantes que la alimentación durante la lactancia son las reservas con que la madre inicia esta etapa, por lo que la asistencia nutricional debe iniciarse durante el embarazo o antes. De esta forma se lograría un mejor crecimiento y desarrollo intrauterino y simultáneamente prolongar una lactancia materna exclusiva en forma efectiva.

La validación de estos resullados puede lograrse a través de programas de suplementación de la embarazada y nodriza. que de demustrar efectus positivos pueden ayudar a reducir los riesgos de salud de las primeras etapas de la vida. Aun cuando el factor nutricional parece importante para asegurar una buena lactancia, obviamente deben considerarse en forma integrada otros aspectos igualmente fundamentiles teducacionales, socioculturales, legales, etc.) para mejorar la dranuatica corta duración de la lactancia en nuestro pais.

\section{RESUMEN}

Con el propósito de estudiar las posibles relaciones entre nutrición y alimentación de la madre, calidad de la leche y crecimiento del lactante se estudiaron 14 nodrizas enflaquecidas y 20 controles durante los 3 primeros meses de lactancia.

El análisis de la dieta por encuesta de tendencia de consumo cuantiticada demuestra un 30.8 y 20.050 de ingesta deficiente en enflaquecidas y controles, respectivamente. Esta cifra sube a $35 \%$ en ambos grupus cuando se considera la encuesta de recordatoriu de 24 horas, pesquisándose además una cifra semejante de madres con ingesta proteica deficiente.

A los 3 meses de lactancia los hijos de madres entlaquecidas presentan signiticativamente menos peso, talla y perimetro braquial que el grupo control. A pesar de la diferencias, en ambos grupos se observaron incrementns comparables con estandares internacionales.

La composición quimica de la leche no demostri diterencias significativas entre controles y enflaquecidas en ninguno de los nutrientes analizados, encontrándose todos ellos dentro de los valores promedios de paises desarrollados.

Al correlacionar el peso del niño a los 3 meses con las variables en estudio, se encontró una correlación signiticativa cun peso de nacimiento, contenido graso, energético y de ácido linoleico de la leche y variables antropométricas de la madre. La cumposición de la leche no se correlacionó con ninguno de los indicadores maternos analizados.

Los resultados sugieren que las diferencias encontradas en el crecimiento de niños de ambus grupos de madres dependen del volumen más que de la composición de la leche. Probablemente sería más importante para asegurar una lactancia exitosa el estado nutricional con que la madre inicia esta etapa, que la alimentación durante este período. Se plantea la necesidad de validar estos hallazgos por medio de estudios de suplementación, que eventualmente podrian reducir los riesgos de morbimortalidad en las primeras etapas de la vida.

\section{REFERENCIAS}

I Jelliffe, D. B., and Jelliffe, E. F. P. The uniqueness of human milk. A in. J. Clin. Nutr. 24: $928,1971$.

2 Harfouche, J. $\boldsymbol{K}$. The importance of Breast-Feeding. J. Trop. Pediat. 16: 135, 1970.

3 Plank, S. G., and Milanesi, M. L. Infant feeding and Infant Mortality in Rural Chile. Bull. WHO 48: 203, 1973. 
4 Margozzint, fR. Lactancia materna en Pediatría. Rev. Chil. Pediatr. 50: 49, 1979.

5 Mata, L. Breast-Feeding: main promoter of infant health. Am. J. Clin. Nutr. 31: 2058, 1978.

6 Mever, H. F. Breast-F eeding in the United States. Clin. Pediatr. 7: 708, 1968.

'Hofvander, Y., and Petros-Baruazian, A. WHO. Collabarative study on Breast-Feeding. Act. Pediat. Scand. 67: 556, 1978.

8 Patri, An y cols. Evaluación de la lactencia materna en América Latína. Pediatría (Santiago) 16: 43, 1973.

9 Patri, A., y Sepúlveda, H. Evolución de la lactancia materna. Pedialría (Santiago) 2l: 2Bl, 1978.

10 Jimenez, J, Alimentación natural del lactante. CONPAN. Sanlìgo, 1976 .

11 Patri, A., y cols. Fomento de la lactancia natural en el mediu eacolar a través de una undad didáctica. Cuad. Méd. Sociales 16 (1): $5,1975$.

12 Ministerio de Salud. Manual de fomento para la lactancia ma Iema. Santiago, 1979.

13 Hanafy, M. M., and Morsey, M. R. A. Maternul Nutrition and Lactation Periormance. J. Trop. Pediat. 18: 18ż, 1972.

14 Thomson, A. M., and Block, A.E. Nutritiongl aspects of human lactation. Bull. WHO 52: 163, 1975 .

15 Jelliffe, D. B, and Jelliffe, E. F. The rolume and composition of human milk in ooorly nourişed communities. Am. J. Clin. Nutr. 31: 492, 1978.

16 Gebre-Medhim, M.; Vahlquist, A.; Hofvander, Y.; Uppsail, L., and Vahlakist, B. Breast milk compusition in Ethiopian and Swedigh mothers. I Vitamin A and H-earotene. Am, J. Clin. Nulr. 29: 441, 1976.

17 Underwood, $B_{+;}$Hepner, R., and Abdulkh, H. Protein, Lipid and Falty acid of Human milk from Pakistani women durine prolonged periods of lactation. Am. J. Clin. Nutr. 23: 400, 1970.

18 Crawford, M. A., Latrance, B. M.; Munhambo, A. E. BreagtFeeding and Human milk composition. The Lancel i: 99, 1977.

19 Deb, A. K, and Cam, H. R. Studies in humen lactation. Brit. J. Nutr, 16: 65, 1962.

20 Mellies, M. J.; Ishikaura, T. T.; Gartside, P. S.; Burton, K, Moc Gee, I,; Allen, K.; Steiner, P. M.; Brody, D., and Gisseck, C. J. Effects of varying materoal dietary fatty acids in lactating wornen and their infants. Am. J. Clin. Nutr. 32: 299, 1979.

21 ICNND. Menual for nutrition surveys. Interdepartmenlal Committee on nutrition for National Defense. Second ed. Bethesda, Maryland, 1963.

22 Jelliffe, $D$. Evaluación del estado de autrición de la comunidad. Serie de Monografian N." 53, OMS, Ginebra, 1968.

23 Graffar, $M$. Une methode de classification sociales d'échantilkns de population. Courrier 6: 455, J956.

24. Deponamenta de Nutriciòn. Fartultad de Medicina Santiago Norte, Universidad de Chile. Técnicas para realizar la encuesta alimentaria por Tendencia de Coneumo Cuantificada. Pub. Doc. 4]9/73.

25 Lechtig, A.; Yarbrough, Ch.; Martorell, R.; Delgado, H., V Kletin, R. The one day recall dietary survey. Arch. Latinoamer. Nulr. 26: 243. 1976.

26 Durnin, A., and Womersiley, J. Bindy fat assessed from total body densily and its estimation from skinfold thickness measurements on 481 men and women aged from 16 to 72 years. Br. J. Nutr. 32: 77, 1974

${ }^{27}$ Hoese Cottlieb. Oficial methuds of andysig of the association of official analytical chemistry. 12th ed. 258, 1975.

29 Markham, A. A. A stean destillation apparutue suitable for microkjeldahl analysis. Biochem. J. 36: 790, 1942.

29 Hawk, Ph. B., et al. Practical Physiolugical chemistry. 13th ed. 573. 1954.
30 Metcalfe, Rapid preparation of fally acid esters from lipids for gas chromatographic anglysis. An. Chem. 38: 514, 1966.

31 Perkin Elmer Corp. Analitical methods Ior atomie absortion spectrophotonnetry. Norwalk, Connecticut. USA, 1969.

32 Dauncey, M. J.; Gandy, G., and Gairdner, D. Assessment of total body fat in infancy froom skinfold thickness meagurements. Arch. Dis. Child. 52: 223, 1977.

33 Pennachiott, I.; Schmidt-Hebbet, H. Tabla de cumpunición quimica de alimentue chilenos. Fac. de Ciencias Químicas, U. de Chile. 5." ed., 1974.

34 INCAP, ICNND. Tabla de composición de alimentus para uso en América Latina, Ed. Interamericana, México, 2." ed.. 1964.

35 FAO/OMS. Vecesidades de Energía y de Pruteinas. Seric de informes técnicos. N. ${ }^{\circ} 522$. Roma, 1973.

36 FAO/OMS. Necesidades de calcio. Serie de informeo técnicos. N. 230 , Roman 1962.

37 Snedecor, G., and Cochran, W. Statistical methods. The lowa State liniversity Press, 6. ${ }^{\mathrm{a}}$ ed., Ames, Iowa, 1972.

38 Atalah, E.; Diaz, E.; Araya,J.; Arteoga, A., y cols. Evaluación nutricional de una población infanto-juvenil del Ares Norte de Santiago. Pediatría (Santiago) 22: 227, $197 \%$.

39 Arteaga, A.; Fondodori, A.; Ltra, P.; Grebe, C., y cols. Caracrerísticas de la alimentación y estado nutritivo de una población de embarazadas del Area Sur Oriente de Santiago. 1974-75. Rev. Med. Chil. 105: 673, 1977.

40 Atalah, $E$., y Bustos, $P$. Relación entre la composición corporal traterna y la ded recièn nacido. Pediatria (Santiago) 22: 117, 1979.

4) Atalah, E., y Bustos, P. Relación entre la nulrición materna y adecuación de la lactsncia. Pediatría (Santiago) 22: 111. 1979.

42 Bustos, $P_{+;}$Hurtado, C., y Ataloh, E. Crecimiento y composición corporal del lactante con alimentaciún natural. XIIt Congreso Nacional de Pediatría. Pucón. Chile. 1979.

4 Enery. W. B.; Conolty, N. L.: Aitchison, J. M.; Dunkley, $W . L$. Inlluence of sampling on fatty acid composition of human milk. Am. J. Clin. Nutr. 31: 1127, 1978.

44 Nelson, W. E. Tratado de Pediatría. W. B. Saunders 6, ${ }^{n}$ ed. Philadelphia. 1976.

45 Fomon, S. Nutrición Infontil. Edit. Interamericana, S.A. 2.* ed. Méxica. 1976.

4h Lonnerdal, B.; Forsum. E.; Hambrets, L. A longitudinal study of the protein, nitrogen and lactose contents of human mill from Swedish well nourished mothers. Am. J. Clin. Nutr. 29: 1127, 1976.

47 Burtado, C. Contenido lipídica y compoeición en ácidos grarus de la leche materns. Tesis Facultad de Ciencias Químicas y Fannacolugicas. U. de Chile, Santiago, 1979.

48 Hall, B. Uniformity of human milk. Am. J. Clin. Nutr. 32; 304, 1929.

49 Arteaga, A.; Lira, P.; Torres, J; Foradori, A, y cols. E.fecto de la alimentación durante el embarazu y del estado nutritivo de lo gestante sobre el recién nacido. Rev. Méd. Chile. 106; 499. 1978.

50 Edozien, C.; Khan, M. A.; Washien, C. I. Protein deficiency in man: results of a Nigerian village study. J. Nutr. 106: 312. 1976.

S1 Lindblad, B. S., and Rahimtoola, A. Y. A pilot study of the quality of humen milk in a lower socie econonic group in Karachi, Pakistan. Acta Pediatr. Scand. 63: 125, 1974.

52 Geissler, C.; Calloway, D. H., and Margen, S. Lactation and pregnancy in Iran. I Diet ond nutritional stalus. Am. J. Clin. Nutr. 31: 341, 1976.

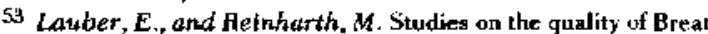
milk during 23 months of lactation in a rural community of the Ivury Coast. Am. J. Clin, Nutr, 32: 1159, 1979. 